\title{
Mechanism Analytical of furcated split of LEFP
}

\author{
Hongwei Liu, Yong Zhang and Junhong Luo
}

Key words: Liner EFP; furcated split; Analytical model

\begin{abstract}
By theoretical analysis, under the conditions of central point initiating, the article consider that furcated split of LEFP was conducted by of every fault plant, $x$ directional speed grads resulted in LEFP's elongation, y directional speed grads resulted in LEFP's bending distortion, thereby result in LEFP's furcated split, and constitute analysis model of furcated split of LEFP, theoretical analysis was in accordance with the experiment, It could provide theoretical basis to defend and dispel furcated split of LEFP.
\end{abstract}

\section{Preface}

Line EFP(Linear Explosive Formation Penetration call LEFP again) usually Be the shot explodes to strike a guided missile target of injure element, cultural heritage model process characteristics to carry on a great deal of experiment research and number calculation to LEFP, in the process of studying in discover, be deep-fried superb lead definitely high, for carry noodles to start to order to explode or explode on middle point, LEFP invades Dip into appear horizontal cent pit to the knothole and put forward LEFP exercises in the air process in at pack medicine length the direction will appear fork crack, and carry on an initial analysis, study to the LEFP fork crack reason.

\section{The LEFP fork splits to analyze model}

\subsection{Basic assumption}

Split mechanism analysis according to the above LEFP fork, with explode on central point of Long for example, make as follows basic assumption as follows:

1 Take no account of to explode to bomb a boundary effect and turn Cape effect influence;

2 The LEFP medicine type covers a horizontal piece the noodles is in the process of exercising in still keep keeping for the flat surface, just shape occurrence variety, after transforming LEFP Zong stalk line is flat surface curve

3 After LEFP forms, neglect same horizontal piece the speed weight of the X, Y direction is inside the noodles variety, namely LEFP horizontal piece the speed weight of the $\mathrm{X}$ inside the noodles, $\mathrm{Y}$ direction is a constant; But follow lengthways, $\mathrm{X}$ inside each cross section and $\mathrm{Y}$ direction of speed weight existence speed gradient.

4 Horizontal piece $\mathrm{X}$ inside the noodles and $\mathrm{Y}$ the speed weight of the direction existence speed gradient respectively creation lengthways stalk to transform and bent transform, take no account of the Ou of mutual to match an effect.

\subsection{The LEFP fork splits to analyze model}

because of rise explode point follow length direction two sides for symmetry pack a medicine, a side that need to start to study to explode point explodes the dissemination and medicine form cover of bombing the wave of inside out sport then. Establish to engrave for the $t$ at this time $=0$.In order to 
taking a post to take an a tiny segment in the medicine type cover $A B$, long $B E \Delta x$, Because the of each cross section exists a speed gradient and establish to carry noodles $\mathrm{A}$ to follow $\mathrm{X}$ and $\mathrm{Y}$ direction speed weight be $V_{x}^{A}$ and $\boldsymbol{V}_{y}^{A}$, The B orders place to side to follow $\mathrm{X}$ and $\mathrm{Y}$ direction speed weight for $\boldsymbol{V}_{x}^{B} \boldsymbol{V}_{y}^{A}$.Because the speed weight of the $\mathrm{Z}$ direction the cross section shape that influence LEFP, split the influence on fork not and greatly, take no account of its influence. Tiny segment at beginning be located in $\mathrm{AB}$ position, after time dt after, the tiny segment exercises $\mathrm{CD}$ position, such as Fig 2 show, then tiny segment elongation quantity $\mathrm{BE}$ :

$$
\Delta 1=\sqrt{(\Delta x+d x)^{2}+(\Delta y)^{2}}-d x
$$

The line contingency increases quantity

$$
\mathrm{d} \varepsilon=\frac{\Delta l}{\mathrm{~d} x}=\frac{\sqrt{(\Delta x+\mathrm{d} x)^{2}+(\Delta y)^{2}}-\mathrm{d} x}{\mathrm{~d} x}
$$

In this formula, $\Delta x=\left.V_{x}^{\prime}\right|_{x} \cdot \mathrm{d} x \cdot \mathrm{d} t, \Delta y=\left.V_{y}^{\prime}\right|_{x} \cdot \mathrm{d} x \cdot \mathrm{d} t$, The generation goes into the last type to invite high level endless a little get:

$$
\mathrm{d} \varepsilon=\left.V_{x}^{\prime}\right|_{x} \cdot \mathrm{d} t
$$

Get stalk to pull to stretch a contingency $\varepsilon$ expression a type for

$$
\varepsilon_{1}=\left.\int_{0}^{t} V_{x}^{\prime}\right|_{X} \cdot d t=\left.V_{x}^{\prime}\right|_{X} t
$$

Under the $\boldsymbol{V}_{y}^{A}$ function, LEFP's medicine form cover follow stalk to also produce flections, follow LEFP stalk to take a tiny segment dx, flections transform of several relation such as Fig2show, fig 2(b) is LEFP horizontal piece noodles, the Y stalk is a symmetry LEFP stalk, the neutral stalk of Z stalk horizontal piece noodles, $\mathrm{d} \theta$ for LEFP horizontal piece face reading to turn Cape,

according to flat surface bent the theory transform several relation, the horizontal piece is inside the noodles arbitrarily the flections at 1:00 contingency BE:
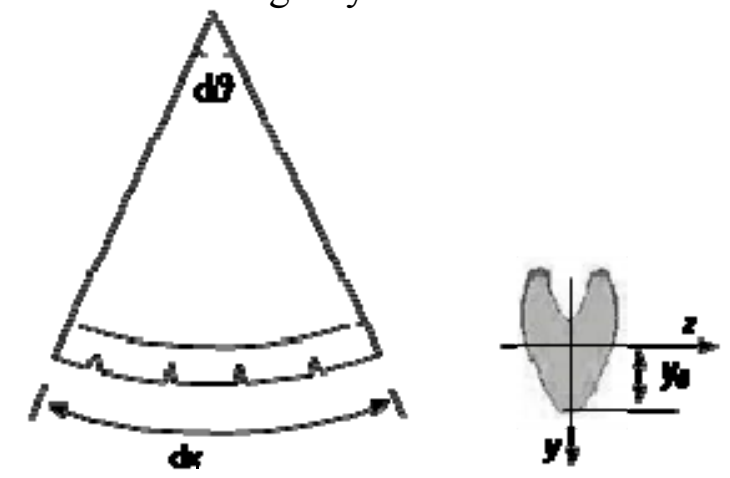

(a) The tiny dollar flections transforms several relationship （b） LEFP Horizontal piece side Fig1 LEFP The tiny dollar flections transforms geometry relationship

$$
\varepsilon_{2}=\frac{\mathrm{d} \theta}{\mathrm{d} x} y_{0}
$$


Explode horizontal piece noodles of LEFP to turn Cape to in the center, with respond that the armor flies plank occurrence to turn Cape to transform likeness. Hen press the relax by taking stroll to explode to bomb to fly plank to turn Cape for:

$$
\theta=\operatorname{arctg}\left(-\frac{V_{y}}{U}\right)
$$

In the type, $U$ for exploded to bomb wave to once sweep plank noodles of speed; ${ }^{V}{ }$ is the vertical that covers a tiny dollar to the speed. key is how beg a tiny dollar of a cover speed is a position function to obviously cover tiny dollar speed, but for medicine type cover same horizontal piece mask tiny dollar but speech, the medicine type covers in the process of quickly shutting to match toward the symmetry side in, the $V_{x} 、 V_{y}$ speed weight is basic Be tended to a stability, can look like ground to use a medicine type of cover coping tiny dollar speed weight to replace the speed weight of the horizontal piece noodles, according to the cultural heritage[5] have:

$$
V_{y}=V_{0} \cos \delta
$$

The LEFP horizontal piece is meeting an emergency into to pull to stretch a contingency and flections contingency inside the noodles and, LEFP horizontal piece the noodles is meeting an emergency into:

$$
\varepsilon=\varepsilon_{1}+\varepsilon_{2}
$$

\section{Experiment verifications and analysis}

Explode to Long middle point at deep-fried is $1000 \%$ and $2000 \%$ cover calibers, LEFP occurrence fork crack. The main reason starts to be ordering to explode to result in a LEFP memory in the speed gradient and exercises to pull to stretch and curve in the process, while deep-frying circumstance, more easily take place fork crack. According to the theory analysis of the front, if adoption long up more order at the same time rise explode can look like to think is a long to be online sex at the same time rise explode, form of LEFP open pit even and tidy. In addition to under the influence of boundary sparse wave outside, compare other two kinds offsets to explode a modeling of way sex, invade effect to be good friends with. But the request ago, difficulty that explodes a way is big, don't easily carry out. Rise to explode at the same time on the arras because of start to need to explode to equip more difficult realization,



Fig2The line raises an explosion $2000 \%$ cover calibers in height experiment knot

\section{Conclusions}

This text passes theory analysis and thinks and explodes LEFP fork and splits to arras central point mainly to is what of each cross section of LEFP existence speed gradient result in, the $\mathrm{X}$ direction speed gradient will make LEFP follow stalk to be pulled and stretch, the Y direction speed gradient 
causes LEFP take place bent transform, result in LEFP take place thus fork crack, and built up a LEFP fork parting to talk about and analyze model, theory with experiment mutually fit together as a result, and split a method to carry on an analytical research to the prevention and the cancellation LEFP fork.

\section{Reference:}

[1] RichardFong, William Ng..Multiple explosively formed penetrator (MEFP) warhead technology development [A].19th International Symposiumon Ballistics [C].Interlaken. Switzerland: 2001, 563-568.

[2] David Bender, Bounmy Chhouk, et al. Explosively Formed Penetrators (EFP) With Canted Fins.19th International Symposium of Ballistics. Interlaken. Switzerland. 7-11 May 2001, 755-762.

[3] A. Helte, et al. A Numerical and Experimental Study of Hemispherical Shaped Charge. 21th International Symposium of Ballistics. 2004. 